\title{
Dielectric elastomer generator with diaphragm configuration
}

\author{
Zhen-Qiang Song ${ }^{1}$, Sriyuttakrai Sathin ${ }^{2}$,Wei Li $^{1}$, Kazuhiro Ohyama ${ }^{2}$ and ShiJie Zhu ${ }^{{ }^{*}}$ \\ ${ }^{1}$ Department of Intelligent Mechanical Engineering, Fukuoka Institute of Technology, Japan \\ ${ }^{2}$ Department of Electrical Engineering, Fukuoka Institute of Technology, Japan
}

\begin{abstract}
The dielectric elastomer generator (VHB 4905, 3M) with diaphragm configuration was investigated with the constant-voltage harvesting scheme in order to investigate its energy harvesting ability. The maximum energy density and energy conversion efficiency is measured to be $65 \mathrm{~J} / \mathrm{kg}$ and $5.7 \%$, respectively. The relatively low efficiency indicates that higher energy conversion efficiency is impeded by the viscosity of the acrylic elastomer, suggesting that higher conversion efficiency with new low-viscosity elastomer should be available.
\end{abstract}

\section{Introduction}

As one kind of electroactive polymers, the dielectric elastomer (DE) possesses some unique properties, such as extraordinarily large strain, excellent flexibility and high response speed [1]. Thus, the DE shows great promise as electromechanical transducer, which is able to convert energy between the mechanical energy and the electrical energy $[2,3]$. When applied as power generators, the DE is able to harvest electricity from various environmental resources, such as the ocean waves, wind, and human activity, which provides a new strategy to acquire clean and renewable source of energy [4, 5]. Compared with other competing power generation technologies, the energy density of dielectric elastomer generator (DEG) shows great advantages, which is more than two orders of magnitude higher than electromagnetics and piezoelectric ceramics [1].

For decades, numerous prototypes adopting different DE materials and various harvesting circuits and schemes have been developed. Huang et al. [6] reported that the energy density of acrylic dielectric elastomer within a single harvesting cycle can be as high as $560 \mathrm{~J} / \mathrm{kg}$ adopting the equi-biaxial stretching method with the constantvoltage energy harvesting strategy. Later, Shian et al. [7] optimized the electromechanical cycle of DEG with a "triangle" harvesting strategy, which boosted the energy density of dielectric elastomer to $780 \mathrm{~J} / \mathrm{kg}$. Even though the maximum energy density is expected to be achieved with equi-biaxial loading strategy in the laboratory, the complex mechanical structure confined its large-scale applications. In contrast, the diaphragm configuration is widely adopted in real applications due to its simple structure and easy installation. However, the research on the diaphragm configuration of DEG with constantvoltage scheme is very few and far between.

In this work, the performance of DEG with diaphragm configuration was investigated adopting the constantvoltage scheme. Then, the energy density and energy conversion efficiency of DEG were analyzed. Finally, strategies for optimizing the performance of diaphragm DEG was discussed.

\section{Experimental methods}

\subsection{Specimen preparation}

The acrylic elastomer VHB 4905 (3M) with a thickness of $0.5 \mathrm{~mm}$ was used for the investigation, and carbon conductive grease was painted on both sides to serve as compliant electrodes. The initial diameter $D_{0}$ of the VHB 4905 film was $12 \mathrm{~cm}$, and then the film was equibiaxially

* Corresponding author: zhu@fit.ac.jp

(C) The Authors, published by EDP Sciences. This is an open access article distributed under the terms of the Creative Commons Attribution License 4.0 (http://creativecommons.org/licenses/by/4.0/). 
pre-stretched by a ratio $\left(D_{1} / D_{0}\right)$ of 2 before attached to the rigid plastic plates. The schematic of the diaphragm specimen is shown in Fig. 1.

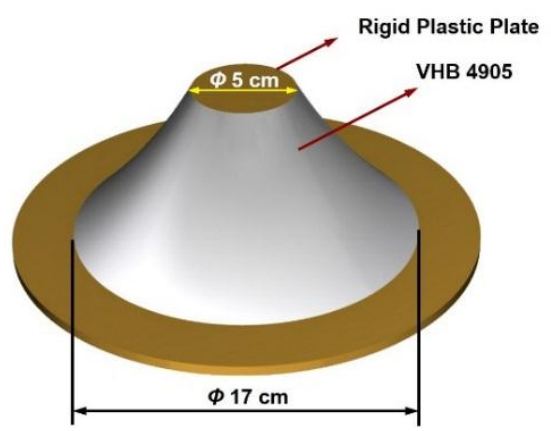

Fig.1 Schematic of the diaphragm specimen.

\subsection{The energy harvesting scheme}

The constant-voltage scheme was adopted in this work. The operational cycles were schematically illustrated on the voltage-charge work-conjugate plane in Fig. 2, and the corresponding electric circuit diagrams is shown in Fig. 3. Twenty Zener diodes with a reverse bias breakdown voltage of $200 \mathrm{~V}$ were connected in series to build a constant harvesting voltage of $4 \mathrm{kV}$.

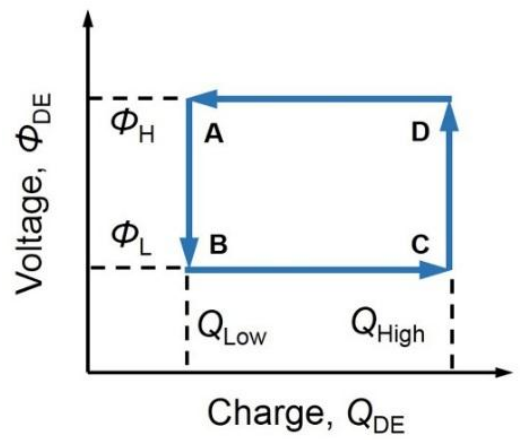

Fig.2 The schematics show the energy-harvesting principle of dielectric elastomer generator.

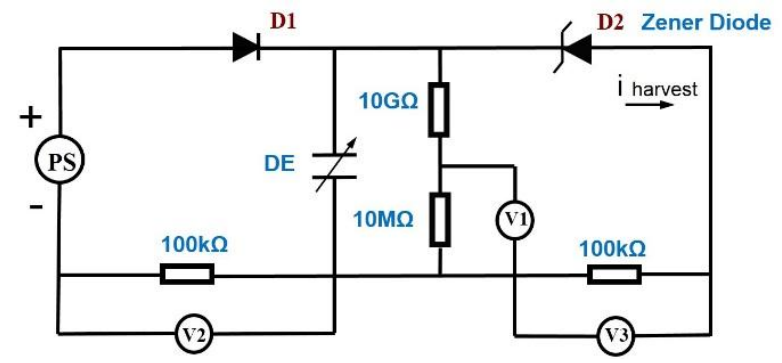

Fig.3 Electrical circuits for energy harvesting.

\subsection{Mechanical loading configuration}

The tests were operated on a self-made testing bench. The stretching of the DE film was accomplished by the motion of a linear servo-motor (GLM20AP, THK) installed with a force sensor (Minebea Co., Ltd.). A multichannel voltmeter (National Instruments) was used to measure the voltages and currents in the circuit. All the data acquisition and the motion of the servo-motors were accomplished with the LabVIEW software.

\section{Results and discussions}

The servo-motor position $(S)$, the force applied by the linear motor $(F)$, the DE voltage $\left(U_{\mathrm{DE}}\right)$, the charging current $\left(i_{\text {charge }}\right)$, and the harvesting current $\left(i_{\text {harvest }}\right)$ versus time $(t)$ were plotted in Fig. 4. The maximum stretch, the input voltage and the harvest voltage were experimentally chosen to be $20 \mathrm{~cm}, 2 \mathrm{kV}$ and $4 \mathrm{kV}$, respectively, which maximized the energy density within a single harvesting cycle while avoiding premature failure of DE, such as mechanical fracture, electric breakdown as well as loss of tension. It can be seen that charge flowed from the power source to the dielectric elastomer membrane when the film was stretched at a constant voltage of $2 \mathrm{kV}$, and then the charge on the dielectric elastomer was harvested when the DE recovered to the initial state at a constant voltage of 4 $\mathrm{kV}$.

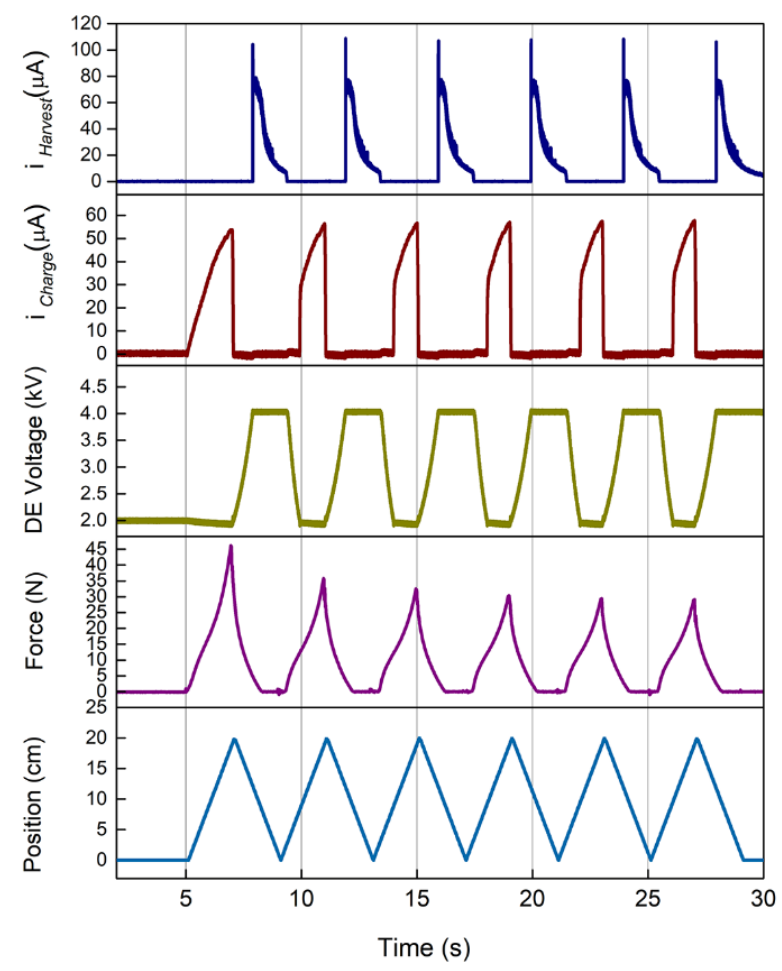

Fig.4 The plot of servo-motor position $(S)$, the force applied by the linear motor $(F)$, the DE voltage $\left(U_{\mathrm{DE}}\right)$, the charging current $\left(i_{\text {charge }}\right)$ and harvesting current $\left(i_{\text {harvest }}\right)$ versus time $(t)$. 


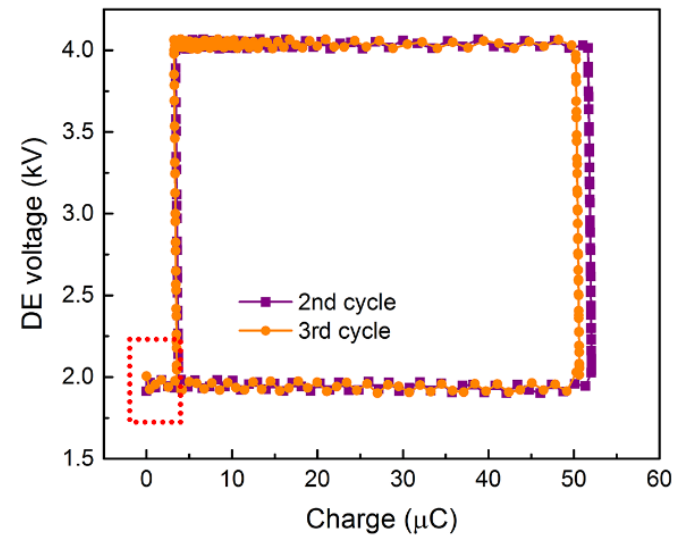

Fig. 5 Voltage of DE as a function of the charge.

The experimental energy harvesting plot, Fig. 5, have the similar basic characteristics as the theoretical scheme on the work-conjugate plane in Fig. 2. The slight distinction is that the charge flowed from the power sources cannot completely harvested during the DE relaxation, as marked by the dotted box in Fig. 5, which is due to the charge leakage within each cycle. The area enclosed by the curve is the net energy gained in a single cycle, $\Delta E_{\mathrm{Net}}$, and the energy density is $E_{\text {Density }}=\Delta E_{\mathrm{Net}} / M$, where $M$ is the mass of the electromechanical active region of the DE membrane coated with carbon grease.

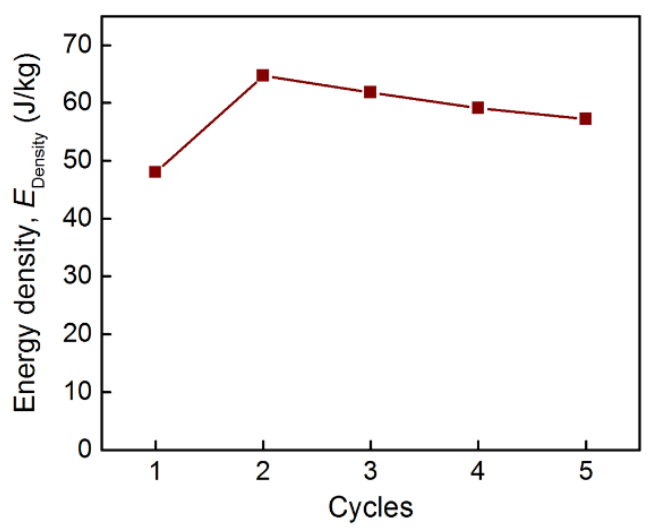

Fig. 6 The energy density within each loading cycle.

The energy density of each cycle is shown in Fig. 6. It can be seen that the average energy density is $58 \pm 6 \mathrm{~J} / \mathrm{kg}$, and the maximum energy density is $65 \mathrm{~J} / \mathrm{kg}$. The corresponding average power and the maximum power is calculated to be $14.5 \pm 1.5 \mathrm{~W} / \mathrm{kg}$ and $16.3 \mathrm{~W} / \mathrm{kg}$, respectively. It should be noted that the power of the DEG should be further improved when the moving velocity of the motor increases. It is found that the energy density decreased after two harvesting cycles, which is due to the loss of tension of DE. It should be noted that in this work, the DE was stretched to approaching its strain limit in order to investigate its maximum energy harvesting ability, while in the actual application with long-term operation, the stretch ratio of the DE was much smaller than its strain limit, and the energy conversion efficiency will not be significantly affected by the increase of the harvesting cycle.

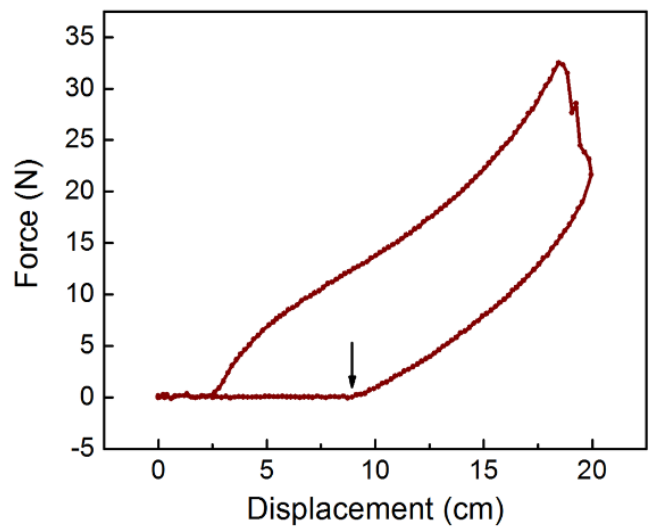

Fig. 7 The force applied by the linear motor versus the servomotor position.

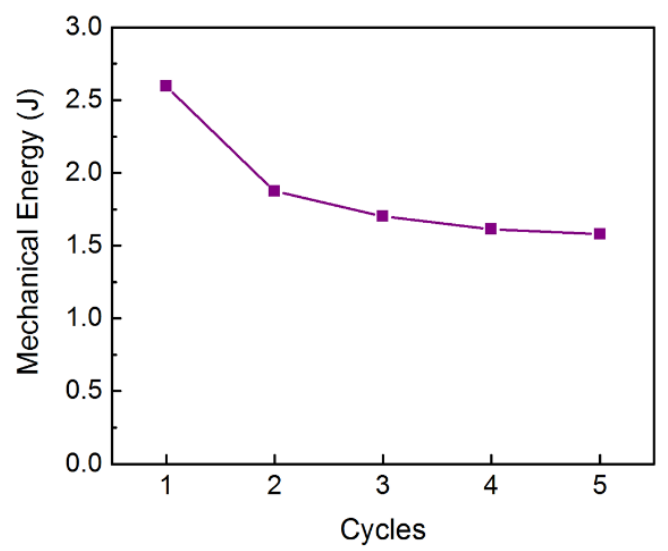

Fig. 8 The mechanical energy dissipation in each cycle.

The relationship between the force applied to stretch dielectric elastomer film and the displacement of linear motor is shown in Fig. 7. It can be seen that during the relaxation process of the $\mathrm{DE}$, the force decreased to be zero when the motor is at the position of $9 \mathrm{~cm}$. Thus, the DE can not relax to the initial state due to the loss of tension within a small motor displacement range, which is caused by the viscosity of the acrylic elastomer. This effect is also evident in Fig. 4, where a delay can be detected between harvesting electric current shut-off and the start of the following harvesting cycle. The mechanical energy dissipation, $\Delta E_{\text {mech, }}$ can be calculated based on the area enclosed by the force-displacement plot in Fig. 7. The mechanical dissipation in each cycle is shown in Fig. 8. It 
can be seen that as the loading cycle increased, the value of $\Delta E_{\text {mech }}$ decreased from $2.6 \mathrm{~J}$ to $1.6 \mathrm{~J}$.

The mechanical-electrical energy conversion efficiency of the DE diaphragm is calculated in terms of the ratio of the net electric energy to the mechanical energy consumed within a single cycle, $\eta=\Delta E_{\mathrm{Net}} / \Delta E_{\mathrm{mech}}$. The value of $\eta$ in each cycle is shown in Fig. 9. It can be seen that the average energy conversion efficiency is $4.8 \pm 1.2 \%$ with the maximum value of $5.7 \%$, which indicated that a large proportion of the mechanical work was consumed via viscous dissipation of acrylic elastomer instead of being transformed into the electrical energy. Therefore, the energy conversion efficiency is expected to be further improved through development of new elastomer materials with low viscosity.

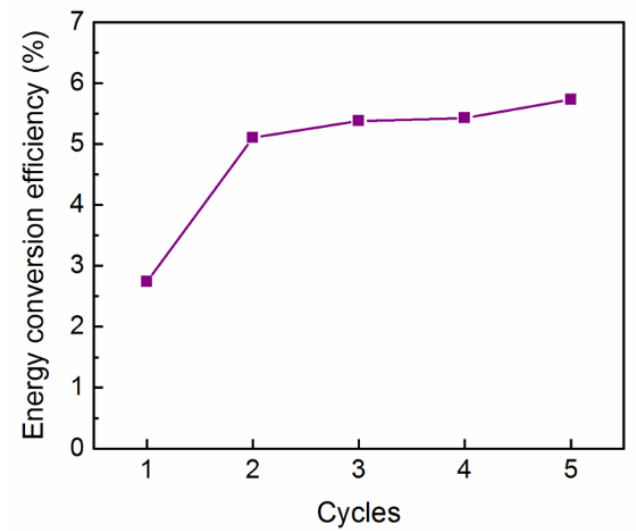

Fig. 9 The energy conversion efficiency in each cycle.

\section{Conclusions}

The dielectric elastomer generator with diaphragm configuration was investigated with the constant-voltage harvesting scheme. The maximum energy density and energy conversion efficiency is measured to be $65 \mathrm{~J} / \mathrm{kg}$ and $5.7 \%$, respectively. After two harvesting cycles, the energy density in a single cycle decreased with the loading cycles, while the energy conversion efficiency increased slightly. The relatively low conversion efficiency indicates that a majority of mechanical energy was consumed by the viscosity of the acrylic dielectric elastomer.

This work was supported by the Strategic Research Foundation Grant-Aided Projects for Private University funded by Japanese Ministry of Education, Culture, Sports, Science and Technology (MEXT).

\section{References}

1. R.D. Kornbluh, R. Pelrine, H. Prahlad, A. Wong-Foy, B. McCoy, S. Kim, et al., MRS Bull. 37, 246-53(2012)

2. R. Pelrine, R. Kornbluh, Q. Pei, J. Joseph, Science 287, 836-9(2000)

3. S. Shian, K. Bertoldi, D.R. Clarke, Adv. Mater. 27, 6814-9(2015)

4. S. Chiba, M. Waki, R. Kornbluh, R. Pelrine, Smart Materials and Structures 20, 124006(2011)

5. K. Ahnert, M. Abel, M. Kollosche, P.J. Jørgensen, G. Kofod, J. Mater. Chem. 21, 14492(2011)

6. J. Huang, S. Shian, Z. Suo, D.R. Clarke, Adv. Funct. Mater. 23, 5056-61(2013)

7. S. Shian, J. Huang, S. Zhu, D.R. Clarke, Adv. Mater. 26, 6617-21(2014) 